\title{
The Importance of Data in the Research of Infectious Diseases
}

\author{
Ojaswita Chaturvedi*
}

Udatta Marketing Pvt Ltd, India

*Corresponding author: Ojaswita Chaturvedi, Udatta Marketing Pvt Ltd, India,

Email: krishnaa.chaturvedi@gmail.com

Short Communication

Volume 2 Issue 2

Received Date: June 20, 2018

Published Date: June 27, 2018
Infectious diseases have been posing severe health hazards and morbidity cases across the globe for quite a few decades now. The perspective cannot be overlooked at any cost when the World Health Organization shows records of about 17 million cases annually in the world. In 2016, there were three communicable diseases among the top ten causes of death. This further emphasizes the harshness of the situation in relation to infectious diseases. Infectious diseases are mostly associated with environmental conditions and there are about 12.6 million cases of death that occur corresponding to environmental factors each year. It is even confirmed by researchers that there are about 30 new emerging infectious diseases over the past 20 years. Following these documentations, it is not surprising that this topic of research has attracted biologists, environmentalists, anthropologists, mathematicians and many other science field workers. The significance of data in any study is very well known as this is what gives bones to the conclusions made. When considering the subject of infectious diseases which is directly linked to human population and morbidity, the inference becomes even more compelling. A handful of infectious disease subdivisions are discussed herewith to highlight the importance of data availability for these researches.

Almost all studies performed on infectious diseases have an aim of either avoiding the outbreak or preventing the spread of these diseases. Infectious disease research can be classified into clinical, epidemiology, pathogenesis, modeling and prevalence, among many others. Considering each area in detail will help in understanding how data plays a crucial role in providing entirety to these issues.

The epidemiology section focuses on emerging diseases, threats of the diseases, disease surveillance, disease detection and vaccination for immunization. Amongst these, surveillance is the collection, analysis and interpretation of disease related information, thus a section that is completely reliable on data. Without sufficient and consistent data, a surveillance report is incomplete. Threats arising from an epidemic can only be analyzed if adequate data is available for scrutinization. The number of cases with respect to appropriate variables helps in successful and correct investigation of the disease. In the case where epidemiology is studied in relation to immigrants or travel, statistics of population movement is also necessary to make any conclusions about the epidemiological status of the disease. Detection studies involve the use of clinical data based on certain geographical divisions to deduce the presence or absence of an epidemic.

Pathogenesis of infectious disease is the detailed study of the respective micro-organism that either causes or transmits the disease. In the context of vector borne diseases, this study is of the vector that is involved in disease transmission. Pathogenesis can be summarized as the mechanism of disease diffusion in a selected population. This subsection includes the life cycle of the pathogen within the environment and/or in the body. Social interaction, environmental changes (weather) or travelling of hosts/infected persons may also effect the entire pathogenesis process. Thus, a detailed data collection that has been stratified into the various aspects that are engaged in the pathogenesis is necessary for a complete study of the pathogen. In order to establish a strong hypothesis in terms of disease communication with respect to the infectious agent, a good set of layered data is a requirement.

Disease prevalence is the resistivity of the disease to any treatment and cure implementations that are applied upon an epidemic outbreak. The persistence of the disease in the environment or in a selected community/geographical location is known as prevalence and is noted for many common infectious diseases. This study is found to be in close similarity to pathogenesis. To provide a strong conclusion about disease prevalence, data over in a significant number of years for the number of cases and/or deaths due to the disease are needed. 
Prevalent nature of a disease can be confirmed only if unswerving and gradual appropriate data is laid out against the regular treatment executions for analysis.

Epidemic modeling is a successful venture which applies mathematical analysis to epidemics giving them a numerical outlook. This assists in quantifying the biological factors which greatly impacts the decisions that can be made for prevention methods. Modeling is sometimes done in the futuristic mode whereby future predictions regarding the transmission of the disease can be obtained. Numerical levels or thresholds of the disease allow the disease to be dealt with in a practical perspective. Here, the disease dynamics are described through mathematical equations. In order to obtain accurate results using modeling, one has to feed in previous data so that the mathematical output matches the practicality of the disease. Without data, assumptions have to be used for modeling and this incurs some significant error levels.

Observing the details described above, it can be seen that infectious disease research cannot be affirmative without the use of suitable data. Studies without the backbone of data will not be able to provide any applicable outputs in terms of disease prevention. Upon evolving through a strong theoretical background, infectious disease studies will only be fruitful in a real world situation once correct data is integrated within. It can be thus concluded that clinical studies is the most important section of infectious disease research. Clinical study is the collection and analysis of disease related information through clinical observations/examinations. This forms the most crucial of all divisions as data is obtained here.

It is unfortunate that as of date, there are numerous data sets that are required for completion of various infectious disease examinations but data availability remains scarce. Disease related data should be carefully collected, stored and made available for all research institutes across the world that are involved in infectious disease research. There are quite a few diseases that are not considered as harmful as others (mainly due to easy treatment or self-cure) and therefore do not have adequate data collection at the clinical centers. Some centers do not expose their clinical data while some countries prefer not to disclose their information openly. It is highly suggested that data should be made available for infectious disease researches to have a holistic direction and their outcomes can be implemented on public and practical platforms.

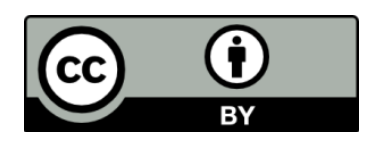

